\title{
Predictores clínicos de mortalidad en el seguimiento a mediano plazo en pacientes adultos inmunocompetentes hospitalizados por neumonía adquirida en la comunidad
}

\author{
FERNANDO SALDÍAS P., FERNANDO ROMÁN O.a, \\ ROLANDO MATURANA O. a, ORLANDO DÍAZ P.
}

\section{Predictors of one year mortality among immunocompetent adults hospitalized for community-acquired pneumonia}

Background: Mortality increases in adults, especially in older adults, after recovery from an episode of community-acquired pneumonia (CAP). Aim: To analyze survival and predictors of death at one year follow up of a cohort of adult patients hospitalized with CAP. Material and Methods: Immunocompetent patients admitted to a clinical hospital for an episode of CAP were included in the study and were assessed according to a standardized protocol. One year mortality after admission was assessed using death records of the National Identification Service. Clinical and laboratory variables measured at hospital admission associated with risk of death at one year follow up were subjected to univariate and multivariate analysis by a logistic regression model. Results: We evaluated 659 patients aged $68 \pm 19$ years, $52 \%$ were male, $77 \%$ had underlying conditions (especially cardiovascular, neurological and respiratory diseases). Mean hospital length of stay was 9 days, $7.1 \%$ died during hospital stay and $15.8 \%$ did so during the year of follow-up. A causal agent was identified in one third of cases. The main pathogens isolated were Streptococcus pneumoniae (12.9\%), Haemophilus influenzae (4.1\%), respiratory viruses $(6.5 \%)$ and Gram-negative bacilli (6.5\%). In multivariate analysis, the clinical variables associated with increased risk of dying during the year of follow-up were older age, chronic neurological disease, malignancies, lack of fever at admission and prolonged hospital length of stay. Conclusions: Age, specific co-morbidities such as chronic neurological disease and cancer, absence of fever at hospital admission and prolonged hospital length of stay were associated with increased risk of dying during the year after admission among adult patients hospitalized with community-acquired pneumonia.

(Rev Med Chile 2013; 141: 143-152).

Key words: Hospitalization; Outcome assessment, health care; Pneumonia; Prognosis; Survival.

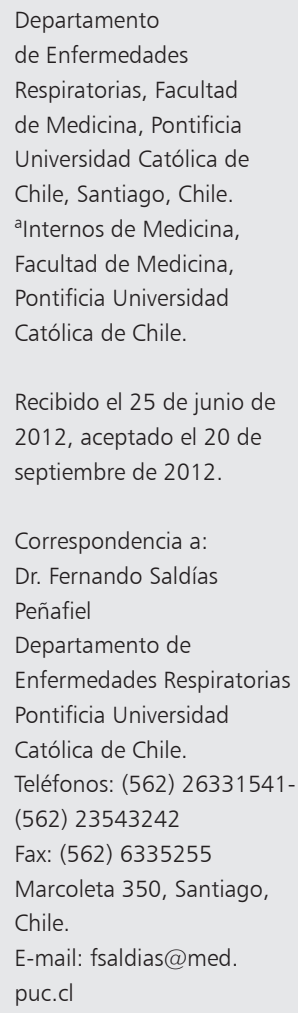

Recibido el 25 de junio de 2012, aceptado el 20 de septiembre de 2012 .

Correspondencia a:

Dr. Fernando Saldías

Peñafiel

Departamento de Enfermedades Respiratorias Pontificia Universidad Católica de Chile. Teléfonos: (562) 26331541 (562) 23543242 Fax: (562) 6335255 Marcoleta 350, Santiago, Chile.

E-mail:fsaldias@med. puc.cl

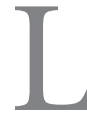
a neumonía adquirida en la comunidad (NAC) es un problema prevalente en la población infantil y adulta de nuestro país, que se asocia a una elevada morbimortalidad, siendo la principal causa de muerte de origen infeccioso ${ }^{1}$. A pesar de la morbilidad y mortalidad considera- blemente elevada asociada a esta condición ${ }^{2,3}$, sólo un número limitado de estudios ha examinado el pronóstico y los factores de riesgo de muerte a largo plazo en los pacientes adultos hospitalizados por neumonía comunitaria ${ }^{4-11}$.

Se ha comunicado una mayor mortalidad en 
la población adulta, especialmente en los adultos mayores, en los años posteriores a la recuperación de un episodio de neumonía ${ }^{4-11}$. Varios estudios han examinado la supervivencia a mediano y largo plazo (1 a 10 años) después del diagnóstico de la neumonía comunitaria; sin embargo, la mayoría de ellos han sido realizados en un solo centro ${ }^{4,11} \mathrm{o}$ el tamaño muestral ha sido reducido ${ }^{4-6,9,11}$; de hecho, sólo un estudio ha incluido pacientes menores de 60 años de manejo ambulatorio en el momento del diagnóstico ${ }^{7}$. Por lo tanto, poco se sabe acerca de la importancia a largo plazo de un episodio de neumonía, especialmente en los adultos jóvenes en etapa productiva.

Aunque los estudios han demostrado claramente que hay una mayor mortalidad a largo plazo después de un episodio de neumonía, no está claro cuáles son las razones y si éstas son modificables. Yende y cols. ${ }^{12}$ comunicaron que la mortalidad a largo plazo era independiente de las comorbilidades del enfermo. Sin embargo, otros autores han demostrado que el aumento en el número de comorbilidades se asocia a mayor riesgo de complicaciones y muerte en el mediano plazo y largo ${ }^{5,7,9}$. CorralesMedina y cols. han comunicado un aumento de las complicaciones cardiovasculares durante y después de una infección grave, como la neumonía, y sugieren que los eventos cardiovasculares podrían jugar un papel importante en el resultado a largo plazo $^{13}$. En un estudio reciente, Mortensen y cols. demostraron que en alrededor de $10 \%$ de los adultos mayores de 65 años hospitalizados por neumonía se diagnosticaba un cáncer pulmonar primario o metastásico en el seguimiento a varios años ${ }^{14}$. Por lo tanto, es probable que otras condiciones médicas y complicaciones sean responsables de al menos parte de este aumento de la mortalidad.

La identificación de poblaciones de riesgo elevado de complicaciones y muerte prematura después del alta hospitalaria nos permitiría implementar medidas preventivas tales como los programas de inmunización, pesquisa precoz de trastornos de la deglución, evaluación y manejo óptimo de las comorbilidades y déficit nutricional. El objetivo de este estudio fue describir la supervivencia a mediano plazo y los factores predictores de muerte en el seguimiento a un año de una cohorte de pacientes adultos inmunocompetentes ingresados en un hospital docente de la Región Metropolitana con el diagnóstico de neumonía adquirida en la comunidad.

\section{Pacientes y Método}

Se evaluaron 659 pacientes adultos inmunocompetentes hospitalizados por un episodio de neumonía adquirida en la comunidad en el Hospital Clínico de la Pontificia Universidad Católica de Chile entre el $1^{\circ}$ de enero de 2003 y el 31 de diciembre de 2005. Se incluyeron en el estudio los pacientes inmunocompetentes mayores de 15 años que cumplieron los criterios diagnósticos de neumonía comunitaria de Fang y cols. ${ }^{15}$, y los criterios de hospitalización sugeridos por la Sociedad Americana de Tórax ${ }^{16}$. Se excluyeron del estudio los pacientes portadores del virus de inmunodeficiencia humana, tumores sólidos o hematológicos en quimioterapia, neutropenia (recuento de leucocitos $<1.000 / \mathrm{mm}^{3}$ ), tratamiento quimioterápico o inmunosupresor en los seis meses previos a la admisión, o tratamiento con dosis $\geq 20 \mathrm{mg} /$ día de prednisona o su equivalente durante por lo menos un mes dentro de los seis meses anteriores a su ingreso y el paciente moribundo, donde el deceso por el episodio de NAC se considera como el evento terminal previsible de su enfermedad crónica de base. El protocolo fue aprobado por el Comité de Ética de la Institución.

En los pacientes ingresados al protocolo se registraron los siguientes antecedentes clínicoepidemiológicos: edad, sexo, comorbilidades, hábito tabáquico y consumo de alcohol, uso de antibióticos previo a la hospitalización, sospecha de aspiración bronquial (definida como cualquier condición clínica subyacente asociado a compromiso del sensorio, pérdida del reflejo tusígeno o alteración de la deglución), cuadro clínico de presentación y la presencia de alguna enfermedad aguda concomitante (insuficiencia cardiaca, arritmia, isquemia miocárdica, asma bronquial o enfermedad pulmonar obstructiva crónica reagudizada). En la enfermedad neurológica crónica se incluyó la enfermedad cerebrovascular con secuela motora, demencia y enfermedad de Parkinson avanzada. Además, se registró el lugar de ingreso (sala, unidad de cuidado intermedio o intensivo), los signos vitales, la saturación arterial medida por oxímetro de pulso y los exámenes de laboratorio solicitados en la admisión al hospital que se han asociado a un curso clínico complicado o mayor mortalidad $^{16-18}$ (hemograma, gases arteriales, función renal, electrolitos plasmáticos, pruebas hepáticas, proteína C reactiva, glicemia, albuminemia), 
y fueron utilizados en el cálculo de los índices de gravedad descritos por Fine y cols. (PSI score) ${ }^{16}$ y la Sociedad Británica de Tórax (CURB-65) ${ }^{18}$.

Se consignaron los resultados de los exámenes microbiológicos (tinción de Gram y cultivo de expectoración o líquido pleural y hemocultivos) y de los estudios inmunológicos solicitados a los pacientes hospitalizados con NAC de acuerdo al criterio clínico del médico tratante (panel de virus respiratorios en el período de otoño-invierno por técnica de inmunofluorescencia directa, inmunoglobulinas G y M de Mycoplasma pneumoniae y Chlamydia pneumoniae por técnica de inmunofluorescencia indirecta y antígeno urinario de Legionella pneumophila serotipo 1 y S. pneumoniae por técnica de enzimoinmunoanálisis).

A todos los pacientes se les realizó una radiografía de tórax PA y L en la admisión al hospital que fue evaluada por un radiólogo del Departamento de Radiología involucrado en el estudio y que desconocía el cuadro clínico de los pacientes. El patrón radiográfico se clasificó como: a) imagen de relleno alveolar, b) patrón intersticial y c) patrón mixto. Se precisó el grado de extensión del compromiso radiográfico en: a) unilobar, b) multilobar ( $\geq 2$ lóbulos) o c) bilateral. Se registró la presencia de derrame pleural, absceso y cavitación.

Durante la estadía en el hospital se consignaron los tratamientos antimicrobianos y la aparición de las siguientes complicaciones: admisión a UCI, uso de ventilación mecánica invasiva, arritmias, insuficiencia cardiaca congestiva, isquemia miocárdica, accidente vascular encefálico, insuficiencia renal aguda, shock séptico, empiema pleural e infección extrapulmonar. La supervivencia en el hospital y en el seguimiento a 30 días después de la admisión se obtuvo de los registros clínicos. La sobrevida a mediano plazo de los 612 pacientes que sobrevivieron a la hospitalización por neumonía fue obtenida del registro civil.

\section{Análisis estadístico}

Los resultados son expresados como valores promedio \pm desviación estándar para las variables medidas en escala numérica y en porcentaje para las medidas en escala nominal. Las variables cualitativas fueron comparadas mediante la prueba de $\chi^{2}$ y las variables continuas con la prueba $t$ de Student. Para ello se utilizaron los programas Epi-Info 6.0 (CDC, Atlanta) y SPSS 7.0 (SPSS Inc., Chicago). Una curva de supervivencia de Kaplan-
Meier fue construida para evaluar el efecto de la admisión por neumonía en la supervivencia del paciente. La prueba log-rank fue utilizada para evaluar diferencias estadísticas en las curvas de supervivencia de diferentes categorías de riesgo (edad, comorbilidad, estadía en el hospital).

Las variables clínicas y de laboratorio medidas en la admisión al hospital asociadas al riesgo de muerte en el seguimiento a 30 días y a un año fueron sometidos a análisis univariado y multivariado en un modelo de regresión logística (modalidad stepwise) que permite el control simultáneo de múltiples factores. De este modo, los parámetros que no agregaron valor predictivo no fueron retenidos en el modelo. Se calcularon los odds ratio e intervalos de confianza (IC) para el 95\%. Las diferencias entre las variables fueron consideradas significativas con un valor de $\mathrm{p}<0,05$.

\section{Resultados}

\section{Características generales de la población}

En el período del estudio, se evaluaron 659 adultos inmunocompetentes hospitalizados por neumonía adquirida en la comunidad. Las características clínicas de la cohorte son descritas en la Tabla 1. La edad promedio fue $68 \pm 19$ años (rango: 16-101), 52\% eran varones, 77\% tenía comorbilidad (especialmente cardiovascular, neurológica y respiratoria crónica), $44 \%$ eran fumadores y $28 \%$ había recibido antibióticos antes del ingreso. El 78\% de los pacientes correspondían a las categorías de riesgo elevado según Fine y cols. ${ }^{16}$, 95\% fueron tratados con cefalosporinas de tercera generación (ceftriaxona o cefotaxima) asociado a macrólidos (25\%), fluoroquinolonas (13\%) y/o antianaerobios (17\%), 23\% fueron manejados en la UCI y $12 \%$ requirió ventilación mecánica invasiva. La estadía promedio en el hospital fue de 9 días (rango: 1-108), un tercio presentó complicaciones médicas durante la evolución, 47 pacientes fallecieron en el hospital $(7,1 \%)$ y 65 en el seguimiento a 30 días $(9,9 \%)$.

\section{Etiología de la neumonía comunitaria}

La etiología fue establecida en 32,5\% de los episodios de neumonía (Tabla 2). Hemocultivos seriados fueron solicitados a 586 pacientes $(89 \%$ de la población), aislando algún patógeno respiratorio en 59 casos (10\%). La tinción de Gram y 


\section{Tabla 1. Características clínicas de los pacientes hospitalizados por neumonía adquirida en la comunidad}

\begin{tabular}{|c|c|}
\hline Variables & $\begin{array}{c}\text { Promedio } \pm \text { DE (rango) } \\
\text { Número }(\%)\end{array}$ \\
\hline$n$ & 659 adultos \\
\hline Edad (años) & $67,5 \pm 18,8(R: 16-101)$ \\
\hline Sexo (M/F) & $342 / 317(52-48 \%)$ \\
\hline Fumadores & $255 / 580(44 \%)$ \\
\hline Lugar de ingreso: Sala-Intermedio-UCI & $397-109-153(60-17-23 \%)$ \\
\hline Uso previo de antibióticos ( $\leq 7$ días) & $176 / 634(27,8 \%)$ \\
\hline Comorbilidad (n, \%) & $506 / 659(76,8 \%)$ \\
\hline Enfermedad cardiovascular & $214(33 \%)$ \\
\hline Diabetes mellitus & $118(18 \%)$ \\
\hline Enfermedad neurológica crónica & $102(16 \%)$ \\
\hline Enfermedad pulmonar obstructiva crónica & $84(13 \%)$ \\
\hline Neoplasia & $65(10 \%)$ \\
\hline Insuficiencia renal crónica & $46(7 \%)$ \\
\hline Enfermedad hepática crónica & $20(3 \%)$ \\
\hline Neumonía multilobar & $172 / 645(26,7 \%)$ \\
\hline Derrame pleural & $138 / 645(21,4 \%)$ \\
\hline Etiología conocida & $214 / 659(32,5 \%)$ \\
\hline Neumonía bacteriémica & $59 / 586(10,1 \%)$ \\
\hline Uso de ventilación mecánica & $77 / 654(11,8 \%)$ \\
\hline Complicaciones en el hospital & $204 / 641(31,8 \%)$ \\
\hline Estadía en el hospital (días) & $9,3 \pm 8,5(\mathrm{R}: 1-108)$ \\
\hline Mortalidad en el hospital & $47 / 659 \quad(7,1 \%)$ \\
\hline Mortalidad a 30 días & $65 / 659 \quad(9,9 \%)$ \\
\hline Mortalidad a 12 meses & $144 / 659(21,9 \%)$ \\
\hline
\end{tabular}

Nota: El número del denominador corresponde a los pacientes con la variable registrada. $\mathrm{M}$ : masculino, $\mathrm{F}$ : femenino, $\mathrm{UCl}$ : Unidad de Cuidados Intensivos.

cultivo de expectoración fueron obtenidos en 441 pacientes (67\% de la población), aislándose algún microorganismo en $26 \%$ de las muestras satisfactorias según los criterios de Murray y cols ${ }^{19}$. Los principales microorganismos identificados en esta cohorte fueron: Streptococcus pneumoniae (12,9\%), Haemophilus influenzae $(4,1 \%)$, virus influenza A y B (3\%), virus parainfluenza 1, 2 y 3 (2,7\%), Staphylococcus aureus (2,6\%), Mycoplasma pneumoniae $(1,4 \%)$ y bacilos Gram negativos entéricos (6,5\%) (Tabla 2). De las 85 cepas aisladas de S. pneumoniae, todas eran susceptibles a penicilina $(\mathrm{CIM} \leq 2 \mathrm{mg} / \mathrm{ml})$ según los puntos de corte establecidos por el Clinical Laboratory Standards Institute (CLSI ${ }^{20}$ para infecciones extra-meníngeas, once cepas eran resistentes a macrólidos y doce a cotrimoxazol. Hubo dos cepas con resistencia de alto grado a cefotaxima. A su vez, de las 27 cepas aisladas de $H$. influenzae, cinco eran productoras de beta-lactamasa $(18,5 \%)$.

\section{Predictores de mortalidad en el seguimiento a corto plazo}

La mortalidad de los pacientes hospitalizados por neumonía comunitaria en el seguimiento a 30 días fue 9,9\% (Tabla 1). Las variables clínicas y de laboratorio asociadas a mayor riesgo de morir en el seguimiento a corto plazo fueron: edad $>65$ años, presencia de comorbilidad, enfermedad cardiovascular y neurológica crónica, sospecha de aspiración, pérdida de la autonomía y postración, presencia de disnea y compromiso de conciencia; ausencia de fiebre, tos, dolor torácico y escalofríos; hipotensión arterial (PA sistólica $<90$ $\mathrm{mmHg}$ ), taquipnea ( $\geq 24 \mathrm{resp} / \mathrm{min})$, acidosis ( $\mathrm{pH}$ arterial $<7,35$ ), hipoxemia $\left(\mathrm{PaO}_{2}<60 \mathrm{mmHg}\right.$ respirando aire ambiente), nitrógeno ureico sérico mayor de $20 \mathrm{mg} / \mathrm{dL}$, recuento de neutrófilos menor de 4.000/ $\mathrm{mm}^{3}$, hipernatremia, hiponatremia, hiperkalemia, hipoalbuminemia $(\leq 3 \mathrm{~g} / \mathrm{dL})$, hiperglicemia $(>130 \mathrm{mg} /$ dL), neumonía multilobar, derrame pleural, categorías de riesgo IV y $\mathrm{V}$ de Fine, CURB-65 $\geq 2$, la admisión a unidades de paciente crítico y el uso de ventilación mecánica.

En el análisis multivariado, las variables asociadas a mayor riesgo de morir en el seguimiento a 30 días fueron: edad avanzada, enfermedad neurológica crónica, ausencia de escalofríos, taquipnea, derrame pleural, nitrógeno ureico sérico elevado, hipoalbuminemia y uso de ventilación mecánica (Tabla 3). 
Predictores clínicos de mortalidad en neumonía adquirida en la comunidad - F. Saldías et al

Tabla 2. Microorganismos aislados en adultos inmunocompetentes hospitalizados por neumonía adquirida en la comunidad

\begin{tabular}{|c|c|c|c|c|c|}
\hline Patógenos & Esputo & Hemocultivos & Líquido pleural & Serología & Antígenos \\
\hline S. pneumoniae & 36 & 47 & 4 & & \\
\hline H. influenzae & 26 & 1 & 1 & & \\
\hline S. aureus & 17 & & & & \\
\hline E. coli & 12 & 4 & & & \\
\hline K. pneumoniae & 5 & 1 & & & \\
\hline$P$. aeruginosa & 8 & & & & \\
\hline M. catarrhalis & 6 & 1 & & & \\
\hline Streptococcus spp & 4 & 4 & 1 & & \\
\hline Enterococcus faecalis & 1 & 1 & & & \\
\hline Virus Influenza A & & & & & 15 \\
\hline Virus Influenza B & & & & & 5 \\
\hline Virus Parainfluenza & & & & & 18 \\
\hline Otros virus respiratorios & & & & & 5 \\
\hline M. pneumoniae & & & & 9 & \\
\hline C. pneumoniae & & & & 5 & \\
\hline L. pneumophila & & & & & 5 \\
\hline Otros bacilos gram (-) & 5 & & 1 & & \\
\hline Polimicrobianos & 29 & & & & \\
\hline Desconocida & $445(67,5 \%)$ & & & & \\
\hline
\end{tabular}

Tabla 3. Variables clínicas asociadas a mortalidad en el seguimiento a corto plazo (30 días) en pacientes adultos inmunocompetentes hospitalizados por neumonía adquirida en la comunidad. Análisis multivariado

\begin{tabular}{|lccccc|}
\hline Factor pronóstico & Coeficiente & Error estándar & Odds ratio & IC 95\% & p \\
\hline Edad (años) & 0,0343 & 0,0134 & 1,04 & $1,01-1,06$ & 0,0106 \\
\hline Enf. neurológica crónica & 1,1638 & 0,3856 & 3,20 & $1,50-6,81$ & 0,0025 \\
\hline Escalofríos & $-1,7601$ & 0,6711 & 0,17 & $0,05-0,64$ & 0,0087 \\
\hline Frecuencia respiratoria & 0,0492 & 0,0215 & 1,05 & $1,01-1,10$ & 0,0224 \\
\hline Derrame pleural & 0,8601 & 0,3677 & 2,36 & $1,15-4,85$ & 0,0194 \\
Nitrógeno ureico sérico & 0,0164 & 0,0083 & 1,02 & $1,01-1,03$ & 0,0497 \\
Albuminemia & $-1,0799$ & 0,3197 & 0,34 & $0,18-0,63$ & 0,0007 \\
Uso de V. mecánica & 2,2199 & 0,4061 & 9,21 & $4,15-20,4$ & $<0,0001$ \\
\hline
\end{tabular}

\section{Predictores de mortalidad en el seguimiento a mediano plazo}

De los 612 pacientes que sobrevivieron a la hospitalización por neumonía, 97 fallecieron en el seguimiento a un año $(15,8 \%)$. Las variables clínicas y de laboratorio asociadas a mayor riesgo de morir en el seguimiento a mediano plazo fueron: edad avanzada, presencia de comorbilidad, enfermedad cardiovascular, respiratoria y neurológica crónica, neoplasia, sospecha de aspiración, pérdida de la autonomía, ausencia de fiebre, escalofríos y dolor torácico en la historia clínica, compromiso 
Predictores clínicos de mortalidad en neumonía adquirida en la comunidad - F. Saldías et al

de conciencia, hipotensión arterial, taquipnea, función renal alterada, leucopenia, proteína C reactiva menor de $24 \mathrm{mg} / \mathrm{dL}$, acidosis, hipoxemia, hipernatremia, hipoalbuminemia, categorías de riesgo elevado de Fine y CURB-65, la admisión a unidades de paciente crítico, desarrollo de complicaciones y estadía prolongada en el hospital (Tabla 4).

El área bajo la curva receptor operador de las variables clínicas asociadas a riesgo de morir

Tabla 4. Variables clínicas asociadas a mortalidad en el mediano plazo (12 meses) en pacientes adultos inmunocompetentes hospitalizados por neumonía adquirida en la comunidad. Análisis univariado

\begin{tabular}{|c|c|c|c|c|c|}
\hline Factores pronósticos & Pacientes & Fallecidos & Odds ratio & IC $95 \%$ & $\mathbf{p}$ \\
\hline Edad $>65$ años & 363 & 81 & 4,18 & $2,4-7,3$ & $<0,0001$ \\
\hline Ingreso a UTIM-UCI & 227 & 45 & 1,58 & $1,1-2,4$ & 0,0398 \\
\hline Comorbilidad & 463 & 92 & 7,14 & $2,8-17,9$ & $<0,0001$ \\
\hline Enfermedad cardiovascular & 191 & 44 & 2,08 & $1,3-3,2$ & 0,0012 \\
\hline EPOC & 78 & 20 & 2,05 & $1,2-3,5$ & 0,0126 \\
\hline Enf. neurológica crónica & 84 & 32 & 4,38 & $2,6-7,3$ & $<0,0001$ \\
\hline Neoplasia & 61 & 29 & 6,44 & $3,6-11,3$ & $<0,0001$ \\
\hline Sospecha de aspiración & 47 & 19 & 4,24 & $2,3-7,9$ & $<0,0001$ \\
\hline Autovalente & 210 & 17 & 0,15 & $0,1-0,3$ & $<0,0001$ \\
\hline Postrado & 18 & 7 & 5,46 & $1,9-15,4$ & 0,0013 \\
\hline Fiebre & 432 & 49 & 0,35 & $0,2-0,5$ & $<0,0001$ \\
\hline Dolor torácico & 131 & 9 & 0,33 & $0,2-0,6$ & 0,0023 \\
\hline Compromiso de conciencia & 108 & 29 & 2,35 & $1,4-3,8$ & 0,0007 \\
\hline Escalofríos & 207 & 19 & 0,42 & $0,2-0,7$ & 0,0016 \\
\hline P. sistólica $\leq 90$ mmHg & 46 & 13 & 2,27 & $1,2-4,4$ & 0,0187 \\
\hline F. respiratoria $\geq 30 x^{\prime}$ & 269 & 53 & 1,66 & $1,1-2,6$ & 0,0291 \\
\hline Temperatura $<37,5^{\circ} \mathrm{C}$ & 280 & 57 & 1,95 & $1,2-3,0$ & 0,0036 \\
\hline Leucocitos $<4.000 / \mathrm{mm}^{3}$ & 14 & 5 & 3,10 & $1,1-9,4$ & 0,0470 \\
\hline Baciliformes $<500 / \mathrm{mm}^{3}$ & 259 & 55 & 2,19 & $1,4-3,4$ & 0,0007 \\
\hline Nitrógeno ureico > 20 mg/dL & 254 & 51 & 1,72 & $1,1-2,6$ & 0,0176 \\
\hline Creatininemia $\geq 1,4 \mathrm{mg} / \mathrm{dL}$ & 140 & 33 & 1,90 & $1,2-3,0$ & 0,0080 \\
\hline Sodio plasma > $145 \mathrm{mEq} / \mathrm{L}$ & 11 & 5 & 4,34 & $1,3-14,5$ & 0,0172 \\
\hline $\mathrm{pH}$ arterial < 7,35 & 30 & 11 & 2,83 & $1,3-6,2$ & 0,0092 \\
\hline $\mathrm{PaO}_{2} / \mathrm{FiO}_{2}<285$ & 270 & 58 & 2,02 & $1,1-3,5$ & 0,0158 \\
\hline $\mathrm{PCR}<24 \mathrm{mg} / \mathrm{dL}$ & 339 & 70 & 3,97 & $2,1-7,7$ & $<0,0001$ \\
\hline Albúmina sérica $<3,5 \mathrm{~g} / \mathrm{dL}$ & 315 & 66 & 2,40 & $1,5-3,9$ & 0,0005 \\
\hline Complicación en hospital & 165 & 34 & 1,60 & $1,1-2,5$ & 0,0490 \\
\hline Índice de Fine > 90 & 348 & 84 & 6,14 & $3,3-11,3$ & $<0,0001$ \\
\hline CURB-65 $\geq 2$ & 369 & 78 & 3,16 & $1,9-5,3$ & $<0,0001$ \\
\hline Estadía en hospital $\geq 7$ días & 329 & 80 & 5,03 & $2,9-8,7$ & $<0,0001$ \\
\hline
\end{tabular}

Nota: UTIM: Unidad de Cuidado Intermedio, UCI: Unidad de Cuidado Intensivo, EPOC: Enfermedad pulmonar obstructiva crónica, P. sistólica: Presión arterial sistólica, F. respiratoria: Frecuencia respiratoria, PCR: Proteína C reactiva sérica. 
Predictores clínicos de mortalidad en neumonía adquirida en la comunidad - F. Saldías et al

Tabla 5. Área bajo la curva receptor operador (AUC) de las variables clínicas asociadas a mortalidad en el mediano plazo (12 meses) en pacientes adultos inmunocompetentes hospitalizados por neumonía adquirida en la comunidad

\begin{tabular}{|c|c|c|c|}
\hline Factor pronóstico & Área bajo la curva & IC $95 \%$ & $\mathbf{p}$ \\
\hline Edad (años) & 0,70 & $0,66-0,74$ & $<0,0001$ \\
\hline Frecuencia respiratoria & 0,59 & $0,55-0,63$ & 0,0042 \\
\hline Temperatura & 0,60 & $0,56-0,64$ & 0,0005 \\
\hline Baciliformes & 0,60 & $0,56-0,64$ & 0,0023 \\
\hline Nitrógeno ureico sérico & 0,59 & $0,55-0,63$ & 0,0057 \\
\hline pH arterial & 0,58 & $0,54-0,63$ & 0,0216 \\
\hline $\mathrm{PaCO}_{2}$ & 0,58 & $0,53-0,63$ & 0,0234 \\
\hline $\mathrm{PaO}_{2} / \mathrm{FiO}_{2}$ & 0,61 & $0,57-0,66$ & 0,0005 \\
\hline Proteína $\mathrm{C}$ reactiva & 0,63 & $0,59-0,67$ & $<0,0001$ \\
\hline Albúmina sérica & 0,63 & $0,59-0,67$ & 0,0001 \\
\hline Índice de Fine (PSI) & 0,76 & $0,72-0,79$ & $<0,0001$ \\
\hline CURB-65 & 0,67 & $0,64-0,71$ & $<0,0001$ \\
\hline Estadía en el hospital & 0,69 & $0,65-0,73$ & $<0,0001$ \\
\hline
\end{tabular}

Tabla 6. Variables clínicas asociadas a mortalidad en el mediano plazo (12 meses) en pacientes adultos inmunocompetentes hospitalizados por neumonía adquirida en la comunidad. Análisis multivariado

\begin{tabular}{|c|c|c|c|c|c|}
\hline Factor pronóstico & Coeficiente & Error estándar & Odds ratio & IC $95 \%$ & $\mathbf{p}$ \\
\hline Edad (años) & 0,0511 & 0,0128 & 1,05 & $1,03-1,08$ & 0,0001 \\
\hline $\begin{array}{l}\text { Enf. neurológica } \\
\text { crónica }\end{array}$ & 1,7162 & 0,3651 & 5,56 & $2,72-11,4$ & $<0,0001$ \\
\hline Neoplasia & 1,7332 & 0,4364 & 5,66 & $2,41-13,3$ & 0,0001 \\
\hline Ausencia de fiebre & 0,6881 & 0,3121 & 1,99 & $1,08-3,67$ & 0,0275 \\
\hline Estadía en el hospital & 0,0626 & 0,0201 & 1,07 & $1,03-1,13$ & 0,0019 \\
\hline
\end{tabular}

en el seguimiento a doce meses se describe en la Tabla 5. En el análisis multivariado, las variables que permanecieron asociadas a mayor riesgo de morir en el seguimiento a un año fueron la edad avanzada, enfermedad neurológica crónica, neoplasia, ausencia de fiebre y estadía prolongada en el hospital (Tabla 6).

En la Figura 1 se representa las curvas de sobrevida de Kaplan-Meier de las principales variables asociadas a mortalidad en el seguimiento a mediano plazo: edad mayor de 65 años, presencia de comorbilidad y estadía prolongada en el hospital.

\section{Discusión}

Los principales hallazgos de este estudio fueron: a) Uno de cada cinco pacientes adultos hospitalizados por neumonía adquirida en la comunidad fallecieron en el seguimiento a un año; b) Los principales agentes causales de neumonía comunitaria fueron S. pneumoniae, $H$. influenzae, virus respiratorios, $S$. aureus y bacilos Gram negativos entéricos; c) Los principales predictores clínicos de mortalidad a corto plazo fueron la edad avanzada, enfermedad neurológica cróni- 


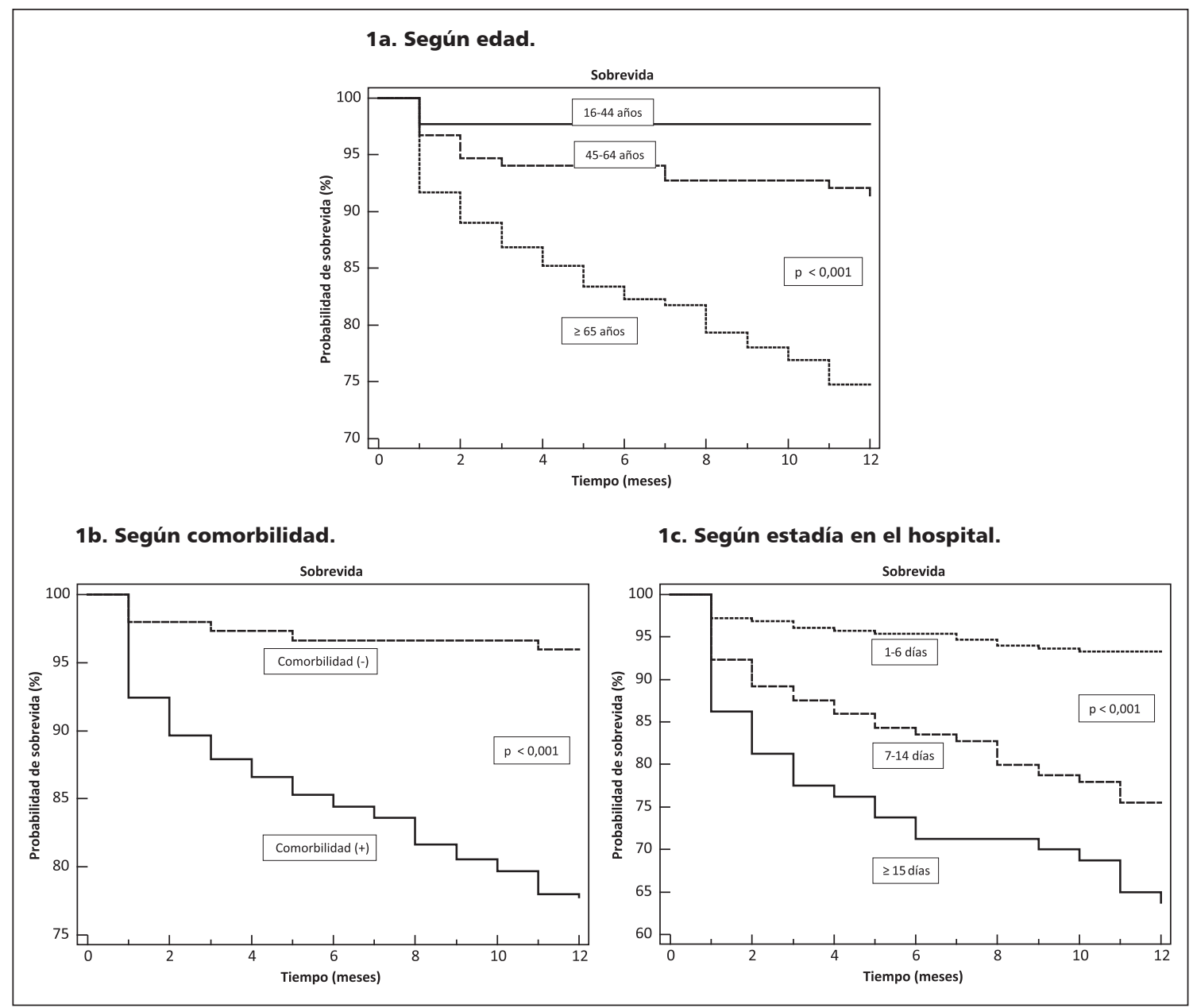

Figura 1. Curvas de sobrevida de Kaplan Meier de pacientes adultos inmunocompetentes hospitalizados por neumonía adquirida en la comunidad según: a) edad; b) comorbilidad y c) estadía en el hospital.

ca, ausencia de escalofríos, taquipnea, derrame pleural, nitrógeno ureico sérico elevado, hipoalbuminemia y uso de ventilación mecánica; d) Las variables clínicas asociadas a riesgo de muerte en el seguimiento a mediano plazo fueron la edad, enfermedad neurológica crónica, neoplasia, ausencia de fiebre y estadía prolongada en el hospital.

Similar a lo descrito por otros autores ${ }^{4-11}$, la edad $^{6,8,9,11}$, las comorbilidades ${ }^{4-6,8,9}$ y las categorías de riesgo elevado de Fine ${ }^{7}$ se han asociado a mayor riesgo de morir en adultos hospitalizados por neumonía comunitaria en el seguimiento a mediano plazo. Brancati y cols. ${ }^{5}$, en una cohorte de 141 pacientes hospitalizados por NAC encontraron que las comorbilidades y el hematocrito menor de 35\% se asociaron a mayor riesgo de muerte en el seguimiento a dos años. Hedlund y cols. ${ }^{4}$, señalaron que el uso de corticoides sistémicos y la presencia de neoplasia se asociaron a mayor riesgo de muerte en un seguimiento a dos años y medio; mientras que la hipoalbuminemia y colonización por bacilos gramnegativos se asoció a mayor riesgo de muerte por neumonía. En una cohorte prospectiva de pacientes adultos hospitalizados y ambulatorios con neumonía comunitaria, Mortensen y cols. ${ }^{7}$ destacaron la capacidad del índice pronóstico de Fine de predecir el riesgo de muerte en el seguimiento a largo plazo (alrededor de seis años); lo cual es explicable por la importancia de la edad y las comorbilidades en el diseño de este índice. 
En 2003, Kaplan y cols. ${ }^{8}$, en una cohorte de 158.960 pacientes adultos mayores de 65 años hospitalizados por neumonía comunitaria encontraron una mortalidad de 33,6\% en el seguimiento a un año y los principales factores asociados al riesgo de muerte en el seguimiento a mediano plazo fueron la edad, presencia de comorbilidad, sexo masculino y raza negra. Similar a lo descrito por Guertler y cols. ${ }^{21}$, en nuestro estudio, la ausencia de fiebre, escalofríos y la proteína $C$ reactiva sérica baja se asociaron a mayor riesgo de morir en el seguimiento a mediano plazo. Al parecer, algunos pacientes que logran sobrevivir al episodio de neumonía no son capaces de montar una respuesta inmune normal, lo cual los predispone a una mayor morbimortalidad en el mediano plazo debido a otras enfermedades infecciosas.

Las principales causa de muerte en el mediano y largo plazo en adultos mayores de 60 años hospitalizados por neumonía comunitaria son las enfermedades cardiovasculares e infecciones respiratorias ${ }^{6}$. Por lo tanto, la identificación de la población de adultos mayores con riesgo elevado de complicaciones y muerte en el mediano plazo, podría permitir implementar medidas preventivas eficaces, como son los programas de vacunación, evaluación del riesgo de broncoaspiración, manejo óptimo de las comorbilidades, promoción del ejercicio físico y apoyo nutricional en sujetos con déficit nutricional.

\section{Referencias}

1. Ministerio de Salud de Chile. Programa de Infecciones Respiratorias Agudas (IRA). Departamento de Estadísticas e Información de Salud, Ministerio de Salud de Chile. (http://www.deis.cl).

2. File TM. The epidemiology of respiratory tract infections. Semin Respir Infect 2000; 15: 184-94.

3. Fine MJ, Smith MA, Carson CA, Mutha SS, Sankey SS, Weissfeld LA, et al. Prognosis and outcomes of patients with community-acquired pneumonia. A meta-analysis. JAMA 1996; 275: 134-41.

4. Hedlund JU, Ortqvist AB, Kalin ME, Granath F. Factors of importance for the long term prognosis after hospital treated pneumonia. Thorax 1993; 48: 785-9.

5. Brancati FL, Chow JW, Wagener MM, Vacarello SJ, Yu VL. Is pneumonia really the old man's friend? Two-year prognosis after community-acquired pneumonia. Lancet 1993 ; 342: 30-3.
6. Koivula I, Sten M, Makela PH. Prognosis after community-acquired pneumonia in the elderly. A populationbased 12-year follow-up study. Arch Intern Med 1999; 159: 1550-5.

7. Mortensen EM, Kapoor WN, Chang CC, Fine MJ. Assessment of mortality after long-term follow-up of patients with community-acquired pneumonia. Clin Infect Dis 2003; 37: 1617-24.

8. Kaplan V, Clermont G, Griffin MF, Kasal J, Watson RS, Linde-Zwirble WT, Angus DC. Pneumonia: still the old man`s friend? Arch Intern Med 2003; 163: 317-23.

9. Waterer GW, Kessler LA, Wunderink RG. Medium-term survival after hospitalization with community-acquired pneumonia. Am J Respir Crit Care Med 2004; 169: 91014.

10. O'Meara ES, White M, Siscovick DS, Lyles MF, Kuller LH. Hospitalization for pneumonia in the Cardiovascular Health Study: incidence, mortality, and influence on longer-term survival. J Am Geriatr Soc 2005; 53: 110816.

11. Cecere LM, Rubenfeld GD, Park DR, Root RK, Goss $\mathrm{CH}$. Long-term survival after hospitalization for community-acquired and healthcare-associated pneumonia. Respiration 2010; 79: 128-36.

12. Yende S, Angus DC, Ali IS, Somes G, Newman AB, Bauer D, et al. Influence of comorbid conditions on long-term mortality after pneumonia in older people. J Am Geriatr Soc 2007; 55: 518-25.

13. Corrales-Medina VF, Madjid M, Musher DM. Role of acute infection in triggering acute coronary syndromes. Lancet Infect Dis 2010; 10: 83-92.

14. Mortensen EM, Copeland LA, Pugh MJ, Fine MJ, Nakashima B, Restrepo MI, et al. Diagnosis of pulmonary malignancy after hospitalization for pneumonia. Am J Med 2010; 123: 66-71.

15. Fang GD, Fine M, Orloff J, Arisumi D, Yu VL, Kapoor $\mathrm{W}$, et al. New emerging etiologies for communityacquired pneumonia with implications for therapy. A prospective multicenter study of 359 cases. Medicine (Baltimore) 1990; 69: 307-16.

16. Fine MJ, Auble TE, Yealy DM, Hanusa BH, Weissfeld LA, Singer DE, et al. A prediction rule to identify lowrisk patients with community-acquired pneumonia. $\mathrm{N}$ Engl J Med 1997; 336: 243-50.

17. Saldías F, Viviani P, Pulgar D, Valenzuela F, Paredes S, Díaz O. Factores pronósticos, evolución y mortalidad en el adulto inmunocompetente hospitalizado por neumonía neumocócica adquirida en la comunidad. Rev Med Chile 2009; 137: 1545-52.

18. Lim WS, Van Der Eerden MM, Laing R, Boersma WG, Karalus N, Town GI, et al. Defining community acqui- 
Predictores clínicos de mortalidad en neumonía adquirida en la comunidad - F. Saldías et al

red pneumonia severity on presentation to hospital: an international derivation and validation study. Thorax 2003; 58: 377-82.

19. Murray PR, Washington JA. Microscopic and bacteriologic analysis of expectorated sputum. Mayo Clin Proc 1975; 50: 339-44.

20. Clinical and Laboratory Standards Institute. Performan- ce standards for Antimicrobial susceptibility testing. Eighteenth informational supplement. M100-S18. CLSI, Wayne, PA. USA, 2008.

21. Guertler C, Wirz B, Christ-Crain M, Zimmerli W, Mueller B, Schuetz P. Inflammatory responses predict longterm mortality risk in community-acquired pneumonia. Eur Respir J 2011; 37: 1439-46. 